\section{Modeling the redundant signals effect by specifying the hazard function}

\author{
HANS COLONIUS \\ Universität Oldenburg \\ Oldenburg, Federal Republic of Germany
}

In the bimodal detection task, the observer must respond as soon as a signal is presented in either of two modalities (e.g., a tone or a flash). On single-signal trials, only one signal is presented; on redundant-signal trials, both signals are presented either simultaneously or with a short stimulus onset asynchrony (SOA). The common empirical finding is that response time (RT) is shorter for redundantsignal trials. A number of sensory processing models have been proposed for this redundant-signals effect (RSE). These models differ in terms of assumptions made with respect to the underlying stochastic mechanisms (e.g., Meijers \& Eijkman, 1977; Nickerson, 1973; Raab, 1962).

Recently, special efforts have been made to derive empirically testable properties from various models (e.g., Colonius, 1986; Diederich \& Colonius, 1987; Gielen, Schmidt, \& Van den Heuvel, 1983; Miller, 1982; Ulrich \& Giray, 1986). In this note, we propose a particular way of dealing with some of these models in an integrated manner by using the concept of a hazard function. The hazard function is very closely related to the probability distribution (see definition below). It specifies, for any point in time, the tendency for the response to occur instantaneously given that it has not yet occurred. It will be shown that formulating models for the RSE in terms of the hazard function clarifies their underlying assumptions and facilitates derivation of their testable properties. This will be demonstrated with reference to a recent paper by Miller (1986), in which a number of different models for the RSE are discussed.

The hazard function, a probabilistic concept originally developed in reliability theory (cf. Barlow \& Proschan, 1975), has become a valuable tool in the analysis of RTs (see, e.g., Bloxom, 1984, 1985; Luce, 1986, p. 13ff; Townsend \& Ashby, 1983).

Definition. Let $T$ be a nonnegative real valued random variable with probability distribution function $F(t)$ such that the corresponding density function $f(t)$ exists. The hazard function is defined by

$$
h(t)=f(t) /(1-F(t))
$$

for all $t$ for which $F(t)<1$.

The author wishes to thank Adele Diederich, Christa Patzelt, and Edmund Schirrmeister for helpful comments on an earlier version of this note. This work was supported by a grant from Deutsche Forschungsgemeinschaft (Co 94/4-1). Address reprint requests or comments to H. Colonius, Institut für Kognitionsforschung, Universität Oldenburg, P.O. Box 2503, D2900 Oldenburg, Federal Republic of Germany.
According to this definition, the hazard function at time $t$ is just the density renormalized by the probability that the response failed to occur prior to $t$, that is, $1-F(t)$. Model assumptions formulated in terms of the hazard function can easily be translated into conditions on the corresponding probability distribution of response times by noting the following relationship (see, e.g., Barlow \& Proschan, 1975, p. 53), which follows from integrating both sides of Equation 1:

$$
F(t)=1-\exp \left[-\int_{o}^{t} h(s) d s\right] .
$$

In the following, we first demonstrate our approach on the well-known independent probability summation model; later, the so-called "exponential coactivation" model proposed by Miller (1986) will be dealt with.

Let $V, A, R$ be the RT if a single visual, a single auditory, and a simultaneous double (redundant) stimulus are presented, respectively. For the corresponding RT probability distribution functions, let us write $F_{V}, F_{A}$, and $F_{R}$. The corresponding hazard rates are denoted $h_{V}, h_{A}$, and $h_{R}$. For the rest of this paper, we assume an experimental situation where a visual stimulus is followed by an auditory stimulus $d$ msec later $(d \geq 0)$. The corresponding probability distribution of RTs is denoted by $F_{R}(t ; d)$ (note that, in particular, $\left.F_{R}(t)=F_{R}(t ; 0)\right)$.

\section{Independent Probability Summation Model}

It is assumed that each stimulus presented generates a neural activity that may trigger the response mechanism. If two stimuli are present, the response is caused by the first process to finish (hence, the name "race models" sometimes used). Thus, in the redundant-signal situation, if the processes do not influence each other, their tendencies to elicit a response instantaneously should add up to an overall tendency to terminate the next instant of time. Taking into account that the acoustic stimulus is presented $d$ msec later, this situation translates into the following hazard function for a redundant-signal trial:

$$
h_{R, d}(t)= \begin{cases}h_{V}(t) & \text { if } t \leq d \\ h_{V}(t)+h_{A}(t-d) & \text { if } t>d .\end{cases}
$$

In order to derive this model's prediction for the distribution of RTs in the redundant-signal situation, we have to insert the above hazard function into Equation 2. This yields, after some simple algebraic manipulation (see Appendix), the familiar expression for the independent probability summation model:

$$
\begin{aligned}
& F_{R}(t ; d)= \\
& \begin{cases}F_{V}(t) & \text { if } t \leq d \\
1-\left[1-F_{V}(t)\right] *\left[1-F_{A}(t-d)\right] & \text { if } t>d .\end{cases}
\end{aligned}
$$




\section{Exponential Coactivation Model}

This class of models proposed by Miller (1986) also assumes a different response tendency for each signal type: acoustic $(A)$ visual $(V)$, or simultaneous acoustic/ visual $(R)$ signal present. The intuition for the exponential model is as follows:

Consider a trial on which a visual signal is presented first and an auditory signal is presented after some nonzero $\mathrm{SOA}_{\mathrm{A}}$. According to exponential models, a response observed on this trial will have been activated either by the visual signal present before the end of the SOA $A_{A}$ or by the redundant signals present after the end of the $\mathrm{SOA}_{\mathrm{A}}$. (Miller, 1986, p. 333)

By a rough analogy with the race model, Miller then came up with the following inequality for the corresponding probability distributions:

$$
F_{R}(t ; d) \leq F_{V}(t)+F_{R}(t-d) \text { for all } t .
$$

It is possible, however, to derive a much stronger relation between these distributions if exponential coactivation is to hold. Obviously, the hazard function for exponential coactivation models reads as follows:

$$
h_{R, d}(t)= \begin{cases}h_{V}(t) & \text { if } t \leq d \\ h_{R}(t-d) & \text { if } t>d .\end{cases}
$$

Note, in particular, that $h_{R, \mathrm{o}}(t)=h_{R}(t)$; inserting the above hazard function into Equation 2 yields (see Appendix)

$$
\begin{aligned}
& F_{R}(t ; d)= \\
& \qquad \begin{array}{ll}
F_{V}(t) & \text { if } t \leq d \\
1-\left[1-F_{V}(d)\right] *\left[1-F_{R}(t-d)\right] & \text { if } t>d .
\end{array}
\end{aligned}
$$

Thus, an explicit expression for the response time distribution of the exponential coactivation model is available and can be used for testing the model. Moreover, since both sides of Equation 7 are probability distributions, standard Kolmogorov-Smirnov tests should be applicable. Of course, Equation 7 implies Miller's Inequality 5.

Exponential coactivation models are history-free in the following sense. By the end of the SOA $(t=d)$, if a response has not yet occurred (triggered by the visual signal alone), the tendency to respond in the next instant of time is reset to the level corresponding to a simultaneous redundant-signal trial, that is, with an SOA equal to zero. Miller (1986) found response times too fast to satisfy Inequality 5 , and was thus led to consider models assuming accumulation of activation over time, as proposed by Grice, Canham, and Boroughs (1984). In their model, response activation grows deterministically as a function of time since onset of the signal. When response activation reaches a criterion, the response is initiated. Variation in $\mathrm{RT}$ is caused by the fluctuation of this response criterion, which is assumed to have a normal distribution with unknown mean and variance. Although derivation of the hazard function-or of closely related quantities-is pos- sible for this type of model too, it does not seem to lead to any simple predictions that would allow a feasible test of the model (see, e.g., Luce, 1986, p. 151).

\section{REFERENCES}

Barlow, R. E., Proschan, F. (1975). Statistical theory of reliability and life testing. New York: Holt, Rinehart \& Winston.

BLохом, B. (1984). Estimating response time hazard functions; An exposition and extension. Joumal of Mathematical Psychology, 28, $401-420$.

BLoxom, B. (1985). Considerations in psychometric modeling of response time. Psychometrika, 50, 383-397.

Colonius, H. (1986). Measuring channel dependence in separate activation models. Perception \& Psychophysics, 40, 251-255.

Diederich, A., CoLonius, H. (1987). Intersensory facilitation in the motor component? A reaction time analysis. Psychological Research, 49, 23-29.

Gielen, S. C. A. M., Schmidt, R. A., \& Van den Heuvel, P. J. M. (1983). On the nature of intersensory facilitation of reaction time. Perception \& Psychophysics, 34, 161-168.

Grice, G. R., Canham, L., \& Boroughs, J. M. (1984). Combination rules for redundant information in reaction time tasks with divided attention. Perception \& Psychophysics, 35, 451-463.

LUCE, R. D. (1986). Response times. Their role in inferring elementary mental organization. New York: Oxford University Press.

MeiJers, L. M. M., \& Eijkman, E. G. J. (1977). Distributions of simple RT with single and double stimuli. Perception \& Psychophysics, 22, 41-48.

MilleR, J. O. (1982). Divided attention: Evidence for coactivation with redundant signals. Cognitive Psychology, 14, 247-279.

Miller, J. O. (1986). Timecourse of coactivation in bimodal divided attention. Perception \& Psychophysics, 40, 331-343.

NICKERSON, R. S. (1973). Intersensory facilitation of reaction time: Energy summation or preparation enhancement? Psychological Review, 80, 489-509.

RAAB, D. (1962). Statistical facilitation of simple reaction times. Transactions of the New York Academy of Sciences, 24, 574-590.

Townsend, J. T., \& AshBY, F. G. (1983). Stochastic modeling of elementary psychological processes. Cambridge: Cambridge University Press.

ULRICH, R., \& GIRAY, M. (1986). Separate-activation models with variable base times: Testability and checking of cross-channel dependency. Perception \& Psychophysics, 39, 248-254.

\section{APPENDIX}

The independent probability summation model is defined by the hazard rate

$$
h_{R, d}(t)= \begin{cases}h_{V}(t) & \text { if } t \leq d \\ h_{V}(t)+h_{A}(t-d) & \text { if } t>d .\end{cases}
$$

It follows from Equation 2 that

$$
1-F_{R}(t ; d)=\exp \left[-\int_{o}^{t} h_{R, d}(s) d s\right] ;
$$

Inserting Equation 3 implies, for $t>d$,

$$
\begin{aligned}
& 1-F_{R}(t ; d) \\
& =\exp -\left[\int_{o}^{d} h_{V}(s) d s+\int_{d}^{t}\left(h_{V}(s)+h_{A}(s-d)\right) d s\right] \\
& =\exp -\left[\int_{o}^{t} h_{V}(s) d s+\int_{d}^{t} h_{A}(s-d) d s\right]
\end{aligned}
$$


a change of variables in the last integral, $s^{\prime}=s-d$, yields

$$
\begin{aligned}
& =\exp -\left[\int_{o}^{t} h_{V}(s) d s+\int_{o}^{t-d}\left(h_{A}\left(s^{\prime}\right) d s^{\prime}\right]\right. \\
& =\left[1-F_{V}(t)\right] *\left[1-F_{A}(t-d)\right] .
\end{aligned}
$$

The exponential coactivation model is defined by the hazard rate

$$
h_{R, d}(t)= \begin{cases}h_{V}(t) & \text { if } t \leq d \\ h_{R}(t-d) & \text { if } t>d .\end{cases}
$$

It follows from Equation 2 that

$$
1-F_{R}(t ; d)=\exp \left[-\int_{o}^{t} h_{R, d}(s) d s\right]
$$

Inserting Equation 6 yields for, $t \leq d$,

$$
\begin{aligned}
1-F_{R}(t ; d) & =\exp \left[-\int_{o}^{t} h_{V}(s) d s\right] ; \\
F_{R}(t ; d) & =F_{V}(t) .
\end{aligned}
$$

Inserting Equation 6 yields, for $t>d$,

$$
1-F_{R}(t ; d)=\exp -\left[\int_{o}^{d} h_{V}(s) d s+\int_{d}^{t} h_{R}(s-d) d s\right]
$$

changing variables, $s^{\prime}=s-d$, in the last integral, we have

$$
\begin{aligned}
1-F_{R}(t ; d) & =\exp -\left[\int_{o}^{d} h_{V}(s) d s+\int_{o}^{t-d} h_{R}\left(s^{\prime}\right) d s\right] \\
& =\left[1-F_{V}(d)\right] *\left[1-F_{R}(t-d)\right] ; \\
F_{R}(t ; d) & =1-\left[1-F_{V}(d] *\left[1-F_{R}(t-d)\right] .\right.
\end{aligned}
$$

(Manuscript received October 7, 1987; accepted for publication October 28, 1987.) 